\title{
Firmicutes-enriched IS1447 represents a group of IS3-family insertion sequences exhibiting unique +1 transcriptional slippage
}

\author{
Ya-Jun Liu' ${ }^{1,2}$, Kuan $\mathrm{Qi}^{1,2,3}$, Jie Zhang ${ }^{1,2,3,4}$, Chao Chen ${ }^{1,2}$, Qiu Cui ${ }^{1,2^{*}}$ and Yingang Feng ${ }^{1,2^{*}}$ (D)
}

\begin{abstract}
Background: Bacterial insertion sequences (ISS) are ubiquitous mobile genetic elements that play important roles in genome plasticity, cell adaptability, and function evolution. ISs of various families and subgroups contain significantly diverse molecular features and functional mechanisms that are not fully understood.

Results: IS 1447 is a member of the widespread IS3 family and was previously detected to have transposing activity in a typical thermophilic and cellulolytic microorganism Clostridium thermocellum. Phylogenetic analysis showed that IS 1447-like elements are widely distributed in Firmicutes and possess unique features in the IS3 family. Therefore, IS1447 may represent a novel subgroup of the IS3 family. Unlike other well-known IS3 subgroups performing programmed -1 translational frameshifting for the expression of the transposase, IS1447 exhibits transcriptional slippage in both the +1 and -1 directions, each with a frequency of $\sim 16 \%$, and only +1 slippage results in full-length and functional transposase. The slippage-prone region of IS 1447 contains a run of nine A nucleotides following a stem-loop structure in mRNA, but mutagenesis analysis indicated that seven of them are sufficient for the observed slippage. Western blot analysis indicated that IS1447 produces three types of transposases with alternative initiations. Furthermore, the IS1447-subgroup elements are abundant in the genomes of several cellulolytic bacteria.

Conclusion: Our result indicated that IS1447 represents a new Firmicutes-enriched subgroup of the IS3 family. The characterization of the novel IS3-family member will enrich our understanding of the transposition behavior of IS elements and may provide insight into developing IS-based mutagenesis tools for thermophiles.
\end{abstract}

Keywords: Transposable element (TE), Frameshift, Lignocellulose, Thermophilic, Clostridium thermocellum

\section{Background}

Transposable elements (TEs) are ubiquitously present in nature [1]. They can change their position in the genome and play critical roles in genome function and evolution [2,3]. The bacterial insertion sequences (ISs) are the simplest TEs that have essential impacts on genome evolution and expression [4]. ISs generally have a length of $0.7-2.5 \mathrm{~Kb}$, containing inverted repeat (IR) sequences at both termini and one or two open reading frames (Orfs) to encode cognate transposase (Tpase) [5]. The

\footnotetext{
*Correspondence: cuiqiu@qibebt.ac.cn; fengyg@qibebt.ac.cn ${ }^{1}$ CAS Key Laboratory of Biofuels, Shandong Provincial Key Laboratory of Energy Genetics, Qingdao Institute of Bioenergy and Bioprocess Technology, Chinese Academy of Sciences, Qingdao, China Full list of author information is available at the end of the article
}

Tpases can recognize the IRs and then catalyze cleavage at the IS ends, followed by IS transfer into the target site via a cut-and-paste or copy-and-paste mechanism. A short flanking, directly repeated (DR) duplication is usually generated at the insertion site during the DNA strand transfer [5-8]. In addition, some IS elements can implement the recoding of their Orfs via a programmed frameshifting strategy at the transcriptional or translational level $[5,9,10]$. In this way, a single DNA fragment can encode different functional Tpases. By controlling the expression intensity and interactions of the Tpases, the transposition activity and specificity of the IS may be regulated [11].

Various ISs are classified into 29 families in the ISfinder Database (https://www-is.biotoul.fr/index.php) 
based on their different transposition chemistry, IR and DR sequence features, Orf organizations, and the nature of their target sequences [13]. The composition and order of the functional domains of the encoded Tpases are also used for IS classification. The IS3 family is one of the largest and best-studied of the IS families and is further divided into 5 main subgroups, including IS2, IS3 [14], IS51 [15], IS407 [16], and IS150 [17], based on the alignment of Orf sequences [13]. The members of the IS3 family have generally conserved IR, terminating with the dinucleotide $5^{\prime}-\mathrm{CA}-3^{\prime}$, and express Tpases via programmed -1 ribosomal frameshifting [5]. The copy-andpaste transposition mechanism of the IS3 family has also been addressed through the extensive analysis of IS911, a member of the IS3 subgroup [8,11, 18-21].

Most of the known IS elements have been derived from mesophilic bacteria rather than thermophiles, according to ISfinder [12]. Identification of thermophilic IS elements has been achieved largely through genomic annotations, rather than from experimental evidence. Although several IS elements have been discovered in thermophilic Caldicellulosiruptor and Clostridium species in the active form, their transposition mechanisms have not been further revealed [22-24]. However, thermophiles are of great interest in industry, because of their unique biochemistry and thermostable enzymes. The importance of thermophilic bacteria in biorefineries has recently been proposed [25]. For example, Clostridium thermocellum (also named Ruminiclostridium thermocellum or Hungateiclostridium thermocellum) is considered a promising biocatalyst in industrial biorefineries for lignocellulosic biomass utilization, and targeted genetic engineering has been widely performed on this cellulolytic and anaerobic thermophile [26-31]. Hence, the detection and functional analysis of thermophilic IS elements may promote greater understanding of the physiology of thermophiles and support the development of thermostable genetic tools.

We discovered an active IS element, IS1447, that could mutate a thymidine kinase (Tdk) gene by insertion when Tdk was used as the counterselection marker during the genomic editing in the $C$. thermocellum DSM1313 strain [26]. IS1447 was also detected in a mutated cipA gene of another C. thermocellum strain ATCC27405 through previous chemical mutagenesis [24]. Genomic analysis revealed fifteen and eighteen copies of the IS1447 element in C. thermocellum DSM1313 and ATCC27405 genomes, respectively. Additionally, sixteen copies of IS1447 were detected in the genomes of two other $C$. thermocellum strains, DSM2360 and AD2, according to recently improved sequencing results [32]. This implied high transposition activity and potential functional importance. IS1447 was annotated as an IS150 subgroup member of the IS3 family [17], but we found unique sequence features and $a+1$ transcriptional slippage pattern in this study. Phylogenetic analysis also showed that IS1447-like elements are widespread in the phylum Firmicutes and not closely related to those from non-Firmicutes species.

\section{Results \\ Discovery of the transposable element IS1447 in C. thermocellum DSM1313}

We previously developed a seamless genome editing system for C. thermocellum DSM1313 using the thymidine kinase gene $t d k$ as a counterselection marker [26]. Theoretically, the Tdk cassette-carrying plasmid should be cured by the host cell in the presence of $10 \mu \mathrm{g} / \mathrm{mL}$ 5-fluoro-2-deoxyuradine (FUDR) because Tdk can convert FUDR to toxic fluoro-dUMP and give rise to cell death by blocking pyrimidine biosynthesis [33, 34]. However, during mutant screening, a majority of the colonies grown on plates with FUDR still contained the transformed plasmid, indicating that Tdk was not functional for counterselection [26].

To confirm the Tdk function during FURD screening, we cultivated the transformants in liquid MJ medium with or without the addition of FUDR continuously through 8 or 3 subculturings, respectively, and then tested the integrity of the $t d k$ gene by colony PCR using primers tdk-F/R (Additional file 1: Table S1). After successive cultivation without FUDR, the size of band was consistent with that of the $t d k$ gene (580 bp), but both 580-bp and $\sim 2-\mathrm{Kb}$ bands were detected when FUDR was present. The $2-\mathrm{Kb}$ band became increasingly dominant along with increased subculturings, and the 580-bp band indicating the wild-type $t d k$ gene was not observed after 3 subcultures (Fig. 1a). Sequencing results suggested that the $t d k$ gene was mutated by the insertion of IS1447, an endogenous TE with a sequence length of $1447 \mathrm{bp}$, which was also detected in C. thermocellum ATCC27405 during previous chemical mutagenesis [24]. Thus, the result confirmed that IS1447 was an active IS element of the thermophilic microorganism.

IS1447 is abundant in the genome of several lignocellulosic species and has unique sequence features

Genome mining showed that C. thermocellum DSM1313 has fifteen IS1447 copies (Clo1313_1104, Clo1313_1298, Clo1313_1865, Clo1313_1651, Clo1313_0773, Clo1313_1935, Clo1313_2369, Clo1313_2700, Clo1313_0507, Clo1313_0508, Clo1313_1640, Clo1313_1641, Clo1313_2656, Clo1313_2663, and Clo1313_2007). Except for Clo1313_2656, which lacks an 8-nt stretch in the middle of the sequence, most of the copies are full-length genes with high sequence identity. 


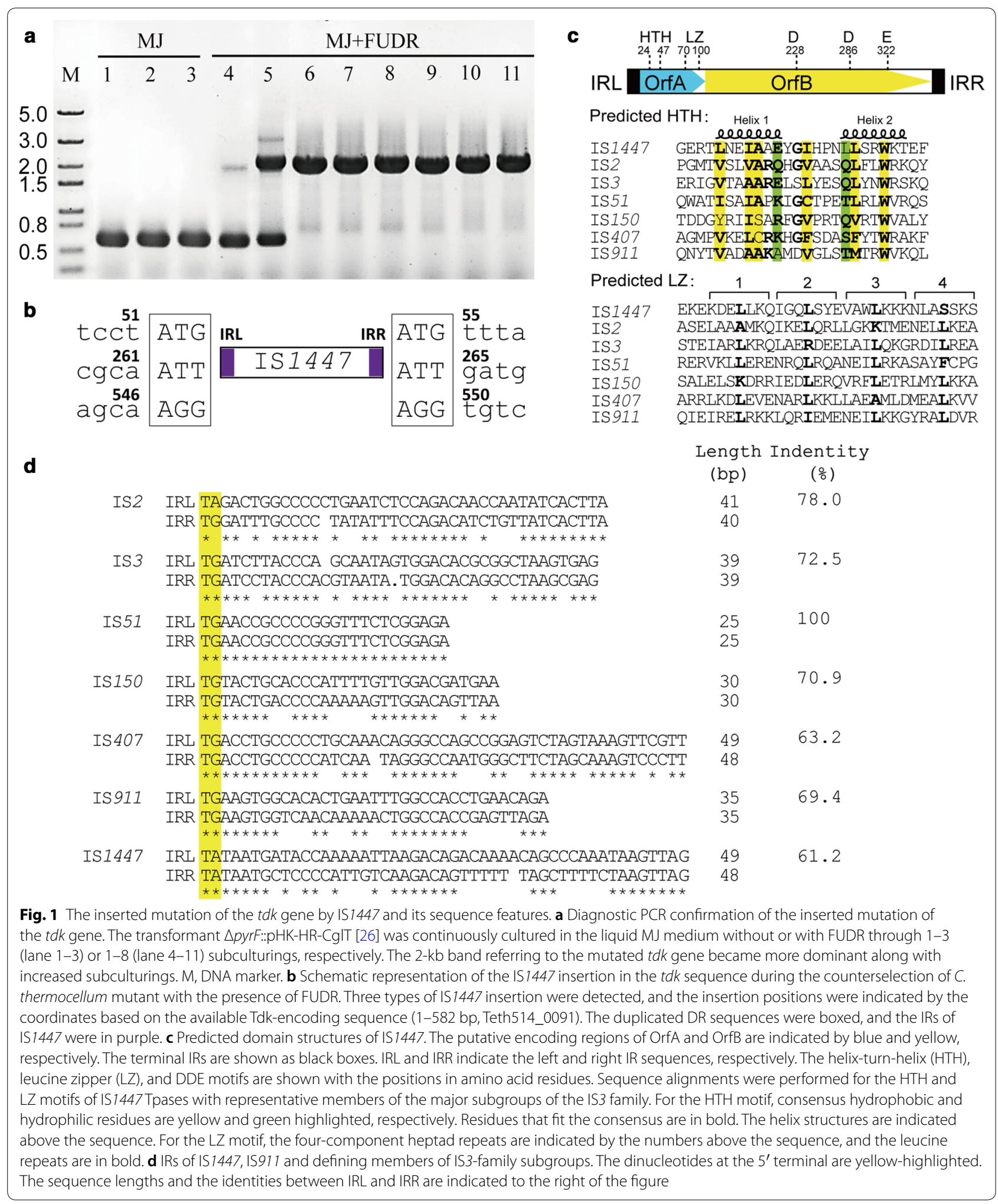


Clo1313_2007 and Clo1313_1640, however, are inserted by Clo1313_2008 and Clo1313_1641, thereby encoding IS256 and another IS1447 Tpase, respectively (Table 1). The sequences upstream and downstream of the IS1447 insertions often appear to be pseudogenes or hypothetical genes (Table 1). This indicated that IS1447 might be involved in the mutation of these once-functional genes. The genomes of $C$. thermocellum strains usually contain multiple IS1447 copies. For example, eighteen IS1447 copies were discovered in the genome of $C$. thermocellum ATCC27405 as previously reported [24]. Sixteen IS1447 copies were found for the genomes of $C$. thermocellum DSM2360 and AD2, the genomes of which have recently been well sequenced and improved [32]. The genotypes of $C$. thermocellum strains can even be differentiated based on the locus and copy numbers of IS1447 [35]. Furthermore, high copy numbers of IS1447-like elements were also detected in the genome of other lignocellulosic species. For example, C. clariflavum ATCC19732 and C. cellulolyticum $\mathrm{H} 10$ are typical thermophilic and mesophilic cellulolytic strains, respectively, while 10 and 11 copies of the IS1447-like element encoding full-length Tpases of 400 and 383 amino acids were detected for them, respectively. In addition, 15, 9, and 4 copies of the IS1447 subgroup ISs were detected during the genomic mining of Clostridium sp. Bc-iso-3, Herbinix hemicellulosilytica DSM $29228^{\mathrm{T}}$ and C. stercorarium subsp.

Table 1 The IS1447 genes and relevant up-
and downstream genes in the $C$. thermocellum DSM1313
genome

\begin{tabular}{|c|c|c|c|}
\hline IS1447 genes & DR & Upstream & Downstream \\
\hline Clo1313_1104 & AAT & Permease & Hypothetical \\
\hline Clo1313_1298 & CAT & Fibronectin & Fibronectin \\
\hline Clo1313_1865 & GAT & Hypothetical & IS256/pseudo \\
\hline Clo1313_1651 & AAC & DNA binding domain & $\begin{array}{l}\text { Hypothetical/crisper } \\
\text { region }\end{array}$ \\
\hline Clo1313_0773 & AAT & Hypothetical & Pseudo/hypothetical \\
\hline Clo1313_1935 & CTG & Pseudo/hypothetical & Pseudo/hypothetical \\
\hline Clo1313_2369 & None & Pseudo/hypothetical & Pseudo/hypothetical \\
\hline Clo1313_2700 & ATT & Pseudo/hypothetical & Pseudo/hypothetical \\
\hline Clo1313_0507 & AAAA & Pseudo/hypothetical & IS3 \\
\hline Clo1313_0508 & GTT & Pseudo/hypothetical & IS3 \\
\hline Clo1313_1640* & AAT & Crisper region & Pseudo \\
\hline Clo1313_1641 & AAAT & Pseudo & Crisper region \\
\hline Clo1313_2656 & CTG & Pseudo/hypothetical & $\begin{array}{l}\text { Pseudo/hypothetical/ } \\
\text { IS116 }\end{array}$ \\
\hline Clo1313_2663 & $\mathrm{CCT}$ & Pseudo/hypothetical & IS4/hypothetical \\
\hline Clo1313_2007* & None & Hypothetical & $\begin{array}{l}\text { ADP-ribosylation/crys- } \\
\text { tallin J1 }\end{array}$ \\
\hline
\end{tabular}

* Clo1313_2007 is inserted by Clo1313_2008 encoding an IS256 transposase in the same direction; Clo1313_1640 is inserted by another IS1447 transposaseencoding gene Clo1313_1641 in the reverse direction stercorarium DSM 8532, respectively, all of which are known cellulose-degrading strains. This result indicated the high abundance and transposition activity of IS1447like elements in several lignocellulosic species [36].

Nucleotide sequence analysis showed that IS1447 is a member of the IS3 family under the name IS120 (https:// www-is.biotoul.fr/index.php) [12]. Like other members of the IS3 family, the IS1447 element is primarily occupied by two consecutive open reading frames (ORF), to code for potential Tpases, which contain the essential structures of a helix-turn-helix (HTH) motif, a leucine zipper (LZ) motif and a DDE domain (Fig. 1c). Additionally, the IS1447 copies in the genome are usually flanked by $3-4$ bp directly repeated duplications (DR), except for Clo1313_2369 and Clo1313_2007, which have no DR sequences (Table 1), and the transposition of IS1447 to the $t d k$ gene also generated 3-bp DR sequences of the target DNA, according to the sequencing results (Fig. 1b). However, IS1447 also presented diverse features from known IS3-family members. According to previous phylogenetic analyses, the predicted OrfA and OrfB proteins of IS1447 are not closely related to any major subgroups of the IS3 family [13]. The IRs of IS1447 have a $5^{\prime}-\mathrm{TA}-3^{\prime}$ dinucleotide at the $5^{\prime}$ terminal instead of the conserved dinucleotide $5^{\prime}$-TG-3' (Fig. 1d). The imperfect IS1447 IRs of different lengths [ 49 and $48 \mathrm{bp}$ for the left (IRL) and right (IRR) IR sequence, respectively] share $61.2 \%$ identity, which is lower than that of known IS3 family IRs (Fig. 1d). An insertion sequence ISPpy1 has similar sequence features with IS1447 in terms of IRs [37], but its evolutionary relationship is not close to IS1447 (see below). This indicated that IS1447 may represent a novel IS3-family subgroup that has a diverse phylogenetic relation with known subgroups.

\section{IS1447 represents a novel subgroup that is Firmicutes-enriched}

To investigate the distribution of IS1447-like elements, BLASTp alignment was performed using the amino acid sequence of IS1447-encoded OrfAB (GenBank Accession Number ADU74917) as the reference. 2111 significant hits were detected with a sequence coverage and identity of over $50 \%$ and $35 \%$, respectively, from 695 organisms (693 from 18 bacterial phyla and 2 archaeal species). Sixty-two sequences were randomly selected to represent different phyla for phylogenetic analysis. The selected OrfAB-like proteins from Firmicutes (28 sequences) and non-Firmicutes (34 sequences) strains were generally separated into two branches of the phylogenetic tree (Fig. 2 and Additional file 1: Figure S1).

IS1447_OrfAB-like Tpases were widespread in the two main Classes, Clostridia, and Bacilli, of the phylum Firmicutes and were also detected in the Classes 


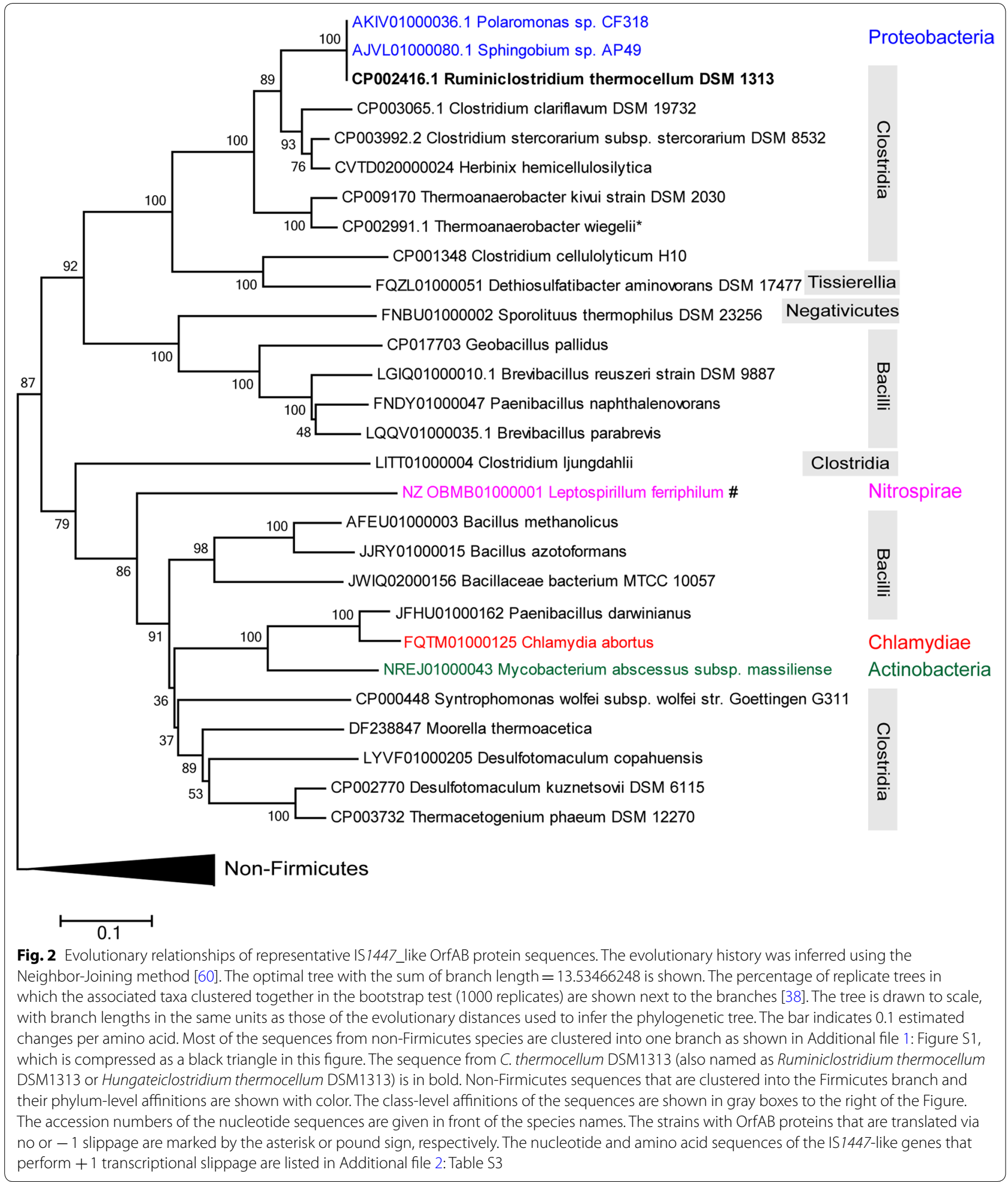

Negativicutes and Tissierellia (Fig. 2). High bootstrap values in the Firmicutes branch indicated closed relationships with IS1447 from C. thermocellum of the IS1447_OrfAB-like Tpases (Fig. 2). Compared to the
Firmicutes branch, the non-Firmicutes branch showed low bootstrap values, indicating low confidence and high variability of the evolutionary relationships [38] (Additional file 1: Figure S1). In addition, nucleotide 
sequence analysis showed that a large proportion (76.5\%, 26 of 34 randomly selected sequences) of the insertion sequences from the non-Firmicutes branch exhibited no or -1 frameshifting for the expression of Tpases, including the ISPpy1 element from the plasmid pKLH80 of Psychrobacter maritimus MR29-12 (GenBank Accession Number AM992204), which was determined previously to display a subgroup of the IS3-family [37] (Additional file 1: Figure S1). Thus, IS1447-like elements are primarily present in the phylum Firmicutes and not closely related to those from non-Firmicutes species. IS1447 can be considered to represent the Firmicutes-enriched insertion sequences belonging to a new subgroup of the IS3 family.

Interestingly, five IS1447_OrfAB-like Tpases from Proteobacteria, Nitrospirae, Chlamydiae, and Actinobacteria were detected in the Firmicutes branch (Fig. 2), in which the ones from the Alphaproteobacteria strain Sphingobium sp. AP49 and Betaproteobacteria strain Polaromonas sp. CF318 had $100 \%$ sequence identity with the IS1447_OrfAB from C. thermocellum DSM1313, while IS1447_OrfAB-like proteins from Chlamydiae and Actinobacteria strains had a close relationship with those from Bacilli strains. This indicated that, in addition to vertical gene evolution, horizontal gene transfer of the IS1447like TEs may also occur among bacterial species.

\section{IS1447 exhibits +1 transcriptional slippage instead of -1 ribosomal frameshifting}

The well-characterized subgroups of the IS3 family generally produce three types of Tpases by -1 frameshifting within a $\mathrm{A}_{6} \mathrm{G} / \mathrm{C} / \mathrm{A}$ motif at the translational level [13]. However, unlike other subgroups, the expression of OrfAB of IS1447 requires +1 frameshift (Fig. 3) [13]. Nucleotide sequence analysis indicated that IS1447 has a run of nine A nucleotides at the $3^{\prime}$ end of the OrfA reading frame, which may be a slippage-prone region for rearrangement at the transcriptional level [39-41].

To investigate whether IS1447 exhibits transcriptional slippage in $C$. thermocellum, the transcript sequences of the potential slippage-prone region were determined by cloning. The total mRNA of $C$. thermocellum DSM1313 was isolated and reverse transcribed to obtain cDNA, which was further used as the template to amplify the IS1447 sequence containing the potential slippage-prone region. The genomic DNA was also isolated and used as the template for PCR using the same primers simultaneously. The PCR

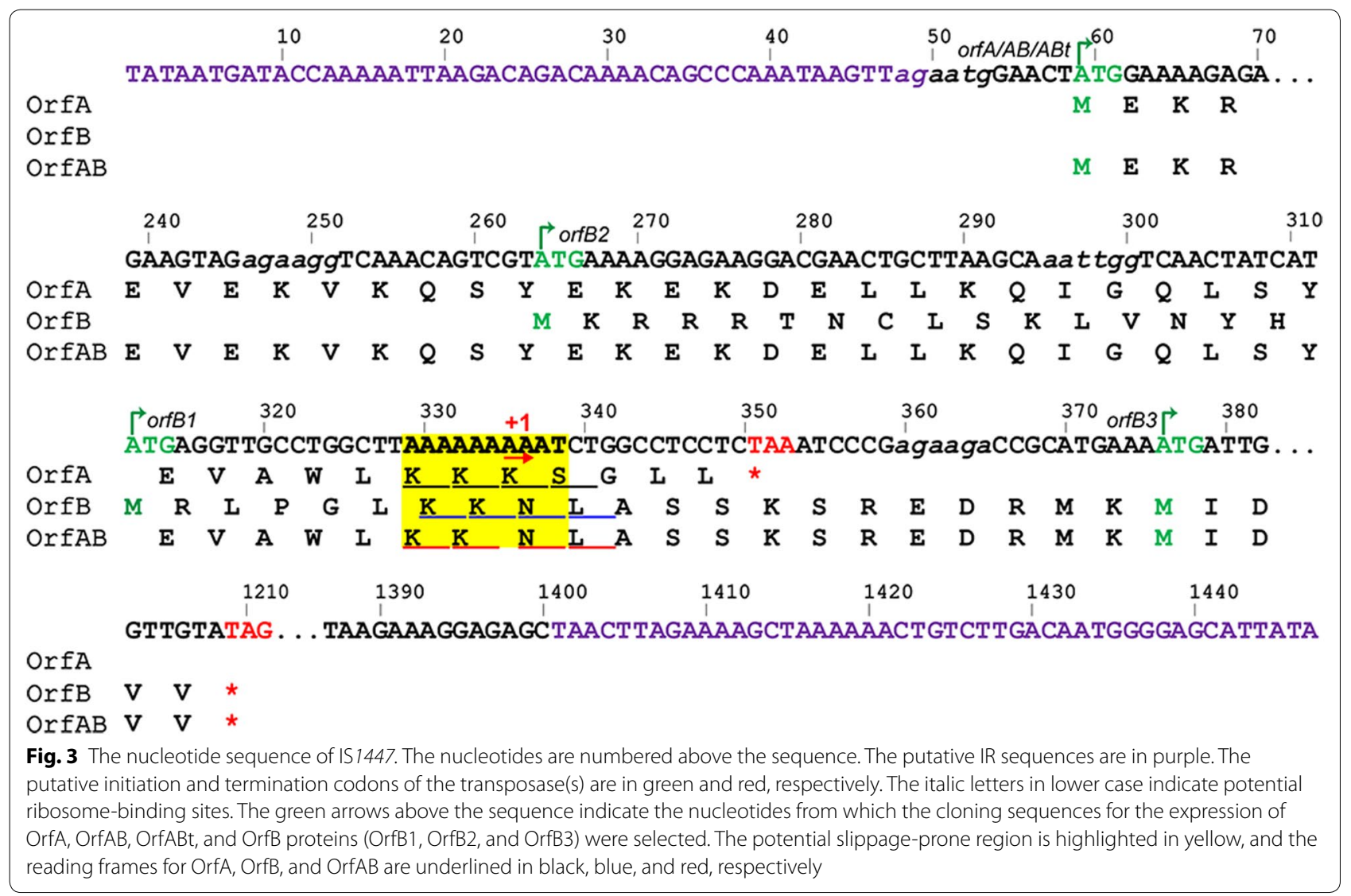


products derived from the cDNA and the control DNA were cloned and sequenced. For the cDNA, 8 and 9 of 52 randomly selected clones had ten and eight A nucleotides in the potential slippage-prone region, respectively, and the other clones showed nine A nucleotides (Additional file 1: Figure S2). In contrast, all 30 clones derived from the control genomic DNA showed nine A nucleotides. The result showed that the IS1447 element exhibits transcriptional rearrangement in both the -1 and +1 directions, while only the +1 transcriptional slippage may result in the expression of a full-length Tpase. The dissociation and reassociation of the nascent RNA with its DNA template within a transcribing RNA polymerase complex may cause transcriptional rearrangement, and the slippage occurred efficiently at the location of homopolymeric runs of A or T nucleotides [40,42]. The dual-direction slippage of IS1447 may be explained by the instability of the A-U rich RNA-DNA hybrid.

\section{IS1447 requires a run of seven A nucleotides for transcriptional slippage}

As shown above, IS1447 produced the fused OrfAB protein via +1 transcriptional slippage within a run of nine A nucleotides (Fig. 3 and Additional file 1: Figure S2). To verify the key region for +1 slippage, various OrfABt mutants were constructed by deleting 3-nucleotide reading frames from the $\mathrm{A}_{9} \mathrm{~T}$ sequence (Fig. $4 \mathrm{a}$ and Additional file 1: Table S2). Immunoblotting analyses showed that both the wild-type OrfABt with the $\mathrm{A}_{9} \mathrm{~T}$ sequence and the positive control OrfABt- $\mathrm{A}_{8}$ produced a His-tagbearing protein of approximately $18.8 \mathrm{kDa}$, indicating the OrfABt protein produced via +1 slippage (Fig. $4 \mathrm{~b}$ ). RNA structure prediction indicated that the mRNA of IS1447 contains a stem-loop structure adjacent to the slippageprone region (Fig. 4c). The mutant carrying a string of seven successive A nucleotides showed the same OrfABt band, indicating that the lack of an AAT sequence did not influence slippage. Other mutants with zero to six A nucleotides showed no band referring to frameshift proteins (Fig. 4b). These results suggested that the +1 slippage of IS1447 required a string containing at least seven

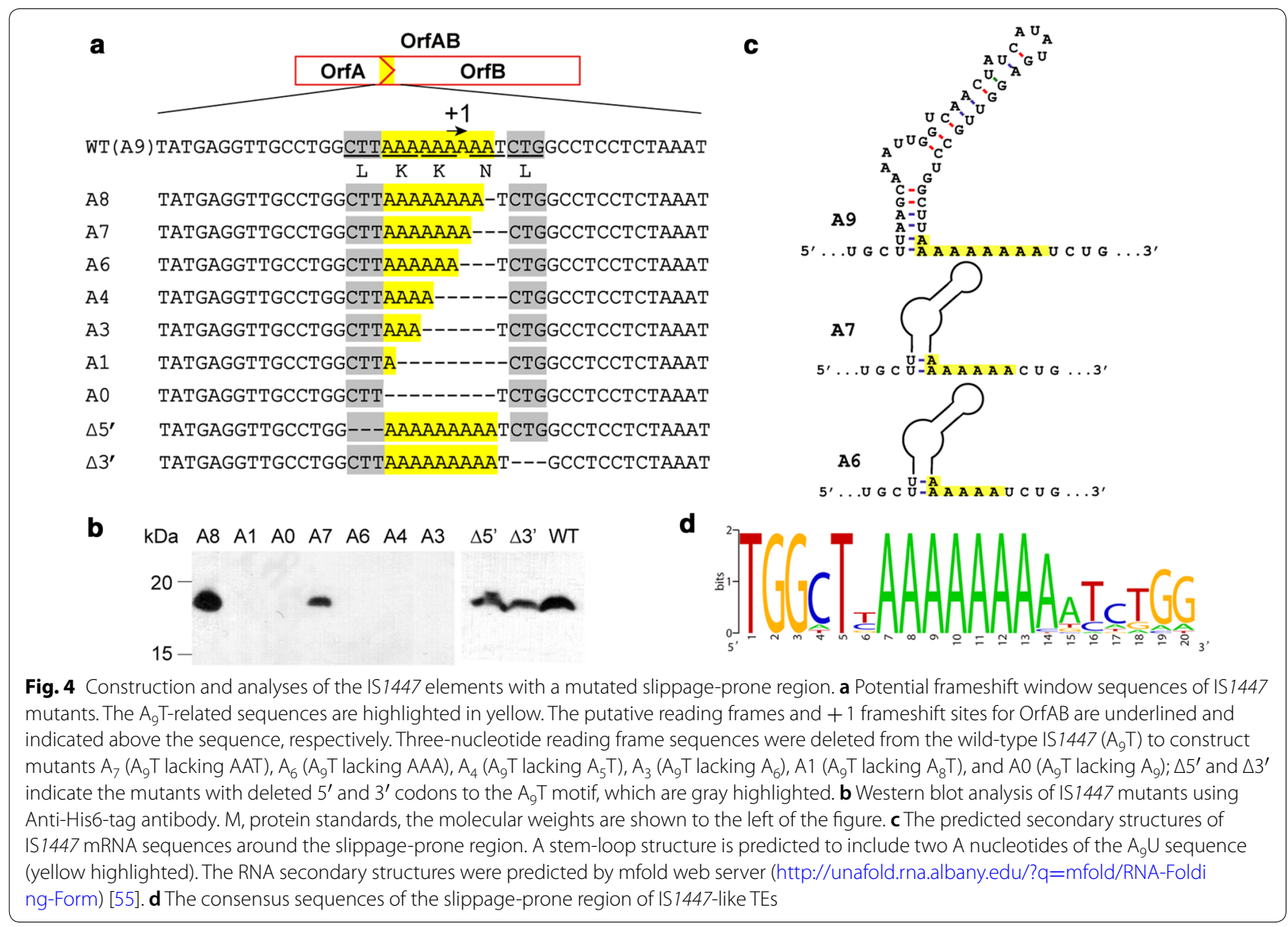


repeated A nucleotides. The slippage-prone sequences of 34 IS1447-like genes (Additional file 2: Table S3) that perform +1 transcriptional slippage were aligned and analyzed by WebLogo [43]. The result indicated that the +1 slippage-prone region had a conserved run of seven to nine A nucleotides (Fig. 4d). Hence, although it has been suggested that the minimum length of the $A$ or $T$ run to promote transcriptional rearrangement is nine [39], IS1447 required only seven A nucleotides for +1 transcriptional slippage. Additionally, a modified stemloop structure could be formed without the $3^{\prime}$ or $5^{\prime}$ codon to the $\mathrm{A}_{9} \mathrm{~T}$ sequence, and the slippage was not apparently influenced (Fig. 4b, c).

\section{IS1447 produces three types of Tpases with alternative initiations}

T7 RNA polymerase-driven expression in E. coli BL21(DE3) was further performed to investigate the production of the IS1447 Tpases via the +1 slippage. The potential ribosome-binding site (RBS) involved in the IRL sequence was used to mimic the protein translation pattern in C. thermocellum (Fig. 3). All produced proteins contained six successive histidines at the C-terminus for affinity purification or immunoblotting (Fig. 5a). Previous studies indicated that IS911, a model for mechanistic analysis of the IS3 family, produced both full-length and truncated OrfAB proteins, and the one lacking the catalytic domain became more abundant at elevated temperature [44]. Hence, a 151-amino acid OrfAB derivate protein truncated for the DDE domain was constructed as well and termed OrfABt. The artificial Tpases OrfAB- $\mathrm{A}_{8}$ and OrfABt- $\mathrm{A}_{8}$ were produced as positive controls for further analyses by deleting a nucleotide $A$ from the $A_{9} T$ string. As shown in Fig. $5 b$ BL21(DE3)::pET21-OrfABt produced a protein of the same size with OrfABt- $\mathrm{A}_{8}$ (approximately $18.8 \mathrm{kDa}$ ). The protein was further verified to be the truncated OrfABt protein by mass spectrometry (Additional file 1: Figure S3). BL21(DE3)::pET21-OrfABt also produced a smaller protein of approximately $11 \mathrm{kDa}$, which may refer to the OrfA protein. This result indicated that the truncated OrfAB could be expressed via +1 slippage in E. coli. However, for the OrfAB-expressing strains BL21(DE3)::pET21-OrfAB and BL21(DE3)::pET21OrfAB- $A_{8}$, only the bands referring to OrfA were observed, and no apparent expression of full-length OrfAB was detected.

The gene encoding an enhanced green fluorescent protein $(e G F P)$ was ligated at the $3^{\prime}$ terminal of the $\operatorname{orf} A B$ sequence to test the expression of the full-length Tpase. The fused protein OrfAB-A $\mathrm{A}_{8}$-eGFP was also expressed as the positive control. Bright green fluorescence was observed for the E. coli strains expressing either OrfAB-eGFP or OrfAB-A -eGFP (Fig. 5c). Because OrfA/OrfAB and OrfB are in the relative translational reading phases 0 and +1 , respectively (Fig. 3), the fused expression of eGFP with OrfB required no slippage. Considering the possibility that the fluorescence is caused by the OrfB-eGFP protein (Fig. 5a), the expressed proteins were also analyzed by Western blotting using the Anti $\mathrm{His}_{6}$-Tag antibody (Fig. 5d). Compared to the positive control OrfAB-A $\mathrm{A}_{8}$-eGFP, a 74-kDa band was clearly detected for BL21(DE3)::pET21-OrfAB-eGFP by immunoblotting but in lower abundance, indicating the successful expression of the fused protein OrfAB-eGFP. Additionally, an $\sim 62-\mathrm{kDa}$ band was detected in high abundance, which is the size expected for a fusion protein OrfB-eGFP (Fig. 5a). These results indicated that the IS1447 produced three types of Tpase, OrfA, OrfB, and the full-length OrfAB, simultaneously.

To define the open reading frames and translation initiation of the IS1447 Tpases, $\mathrm{Ni}^{2+}$-affinity chromatography was employed to purify OrfA, OrfB, and OrfABt proteins for $\mathrm{N}$-terminal sequencing. BL21(DE3)::pET21aOrfA and BL21(DE3)::pET21a-OrfABt were used to express the OrfA and OrfABt proteins, respectively, in which OrfABt was analyzed instead of OrfAB because of the low expression level of OrfAB. $\mathrm{N}$-terminal sequencing showed that OrfA and OrfABt shared the same initiation sequence of "MEKRK," which was consistent with bioinformatics analysis (Fig. 3). Nucleotide sequence analysis showed that OrfB might have three putative initiation codons with potential RBS sequences (Fig. 3). Thus, three E. coli strains, BL21(DE3)::pET21a-OrfB1, -OrfB2, and -OrfB3, were constructed accordingly to express OrfB proteins OrfB1, OrfB2, and OrfB3 with different theoretical molecular weights (M.W.), respectively (Figs. 3 and 5a). However, the OrfB purifications failed because of extremely low (undetectable) expression, and OrfB N-terminal sequencing was not possible. Immunoblotting analysis, however, confirmed that BL21(DE3)::pET21a-OrfB1, -OrfB2, and -OrfB3 produced proteins of $36.9,38.9$, and $34.5 \mathrm{kDa}$, respectively (Fig. 5e), which fit their theoretical molecular weights. Interestingly, all OrfB-expressing strains produced the OrfB3 protein of $34.5 \mathrm{kDa}$ (Fig. 5e). Thus, the ATG at $375 \mathrm{bp}$ of the IS1447 may be the main initiation codon of OrfB (Fig. 3). In this case, the reading frames of OrfA and OrfB have no overlapping region, which is different from known IS3 members that perform frameshifting $[13,19]$. Additionally, a band of approximately $30 \mathrm{kDa}$ was also detected in all OrfB-expressing strains, indicating an alternative initiation codon of OrfB (Fig. 5e). However, no ATG codon was observed downstream of the 375-bp ATG in the IS1447 sequence, and the expression of the 30-kDa protein may be initiated with a non-ATG codon. 


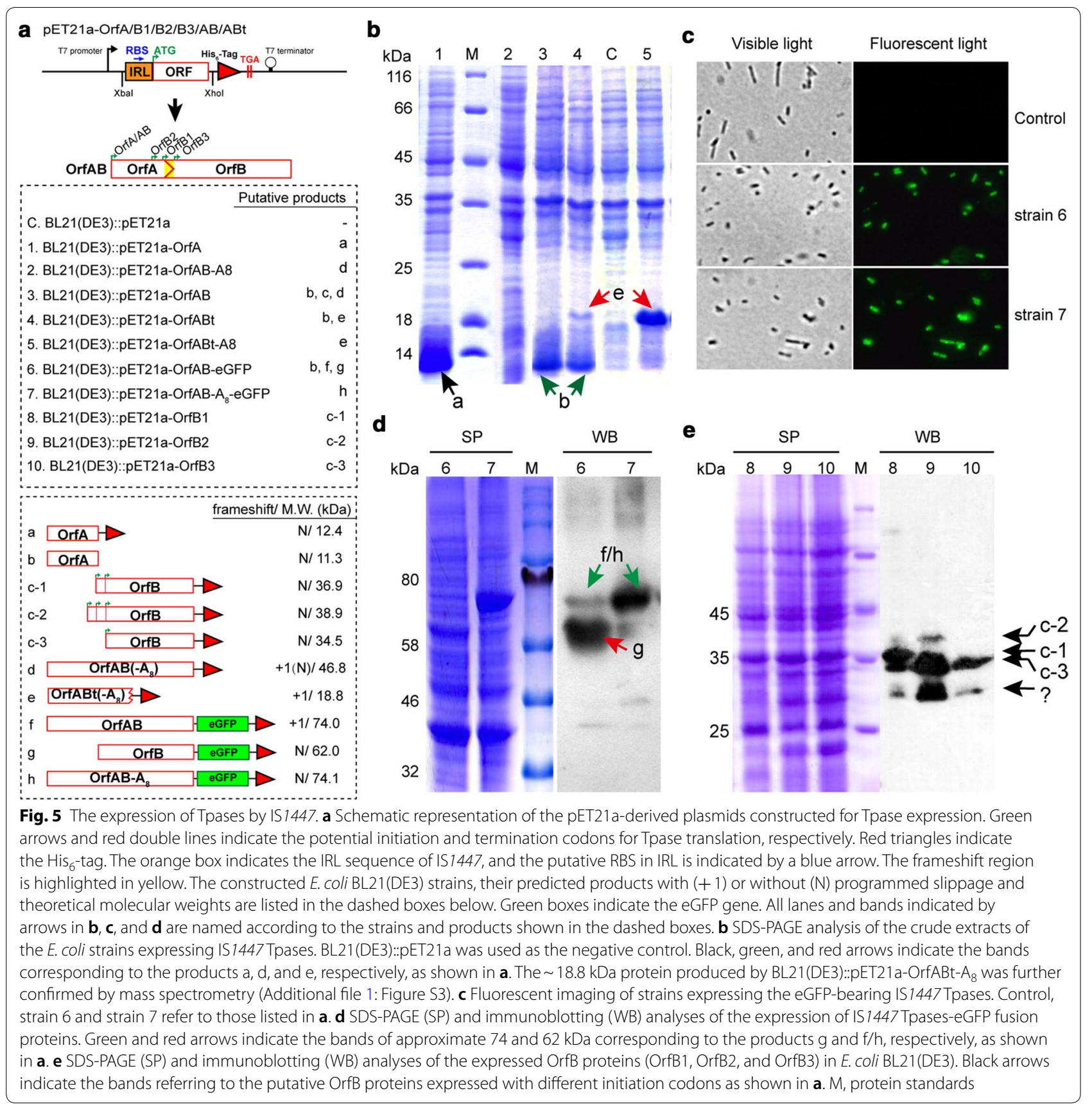

\section{Discussion}

The IS elements are the simplest and most numerous transposable elements that can cause bacterial genome rearrangements and evolution [4, 45]. Certain IS elements have been used to construct transposons for genome characterization, mutagenesis, and editing based on extensive understanding of the sequence features and transposition chemistry [46-49]. However, the IS elements from thermophilic microorganisms are currently underexplored. Several thermophilic IS elements have been reported to have transposition activity, such as ISCbe4, ISCahy1 and IS1447 from Caldicellulosiruptor hydrothermalis, Caldicellulosiruptor bescii, and Clostridium thermocellum, respectively [22-24], which provide insight into developing genetic tools for thermophiles based on endogenous genetic elements, such as the development of the Thermotargetron system for gene targeting based on a thermophilic group II intron [50].

The genomes of the C. thermocellum strains DSM1313, ATCC27405, DSM2360, and AD2 have high IS1447 
copy numbers, indicating the high transposition activity of the IS element. No IS1447 sequence was detected for another three C. thermocellum strains, YS, BC1, and JW20, likely due to the low quality of the genomic sequences at the contig assembly level, since identification of TEs using the current genome sequencing methods is still a challenge [51]. Multiple copies of IS1447-like elements were also detected in several other lignocellulosic species, especially those from phylum Firmicutes. Most of the IS1447-like elements contain relatively conserved long IR sequences that are specific compared to other well-known IS3-family members. IS1447 may not generate double-stranded DNA circles as the well-characterized IS3-family member IS911 [11] because no IRL-IRR junction was detected. Most importantly, IS1447 follows a diverse frameshift pattern of +1 transcriptional slippage compared to other well-known IS3-family members, which read though full-length Orfs using ribosomal frameshifting at the translation level [41, 52]. Evolutionary relationship analysis has shown that IS1447 represents a novel IS3-family subgroup that is Firmicutes-enriched.

Transcriptional rearrangement was first discovered in $E$. coli to produce $\beta$-galactosidase by -1 transcriptional frameshift resulting from the insertion of an extra $A$ [9]. The transcriptional slippage phenomenon has been widely observed since then and has been predicted for IS elements according to genome annotation $[40,41]$. The slippage mechanism has been discussed in previous studies $[39,42,53,54]$. Two main slippage-prone sequence patterns, $X_{m} Y_{n}$ and $A(T)_{n}$, may be involved in bacterial transcriptional realignment. An IS630 family element from a Roseiflexus strain was proved to exhibit transcriptional realignment in the heteropolymeric sequence $\mathrm{T}_{5} \mathrm{C}_{5}$, which fits the $\mathrm{X}_{\mathrm{m}} \mathrm{Y}_{\mathrm{n}}$ pattern [42]. We proved herein that IS1447 employs an $\mathrm{A}(\mathrm{T})_{\mathrm{n}}$ pattern sequence as the slippage-prone region for transcriptional rearrangement. RNA-structure-mediated transcriptional slippage has been proposed for the Roseiflexus-IS630. The hairpin sequence upstream of the $\mathrm{T}_{5} \mathrm{C}_{5}$ slippage-prone region of IS630 is important for slippage [42]. As predicted by the mfold web server [55], the mRNA of IS1447 also contains a stem-loop structure adjacent to the $\mathrm{A}_{9} \mathrm{U}$ region (Fig. 4c), which may play a key role in melting the upstream part of the RNA-DNA hybrid and promoting slippage [42]. Interestingly, two A nucleotides of the $\mathrm{A}_{9} \mathrm{U}$ sequence are involved in the stem-loop structure, which may result in the remaining seven A nucleotide sequences acting as the "true" slippage region. We have proved that the deletion of $\mathrm{AAT}$ of the $\mathrm{A}_{9} \mathrm{~T}$ sequence showed no significant influence but that further replacement of the seventh A with $\mathrm{T}$ completely disrupted the slippage event (Fig. 4b), indicating that the slippage would occur with a run of no less than seven successive A nucleotides. According to previous studies, a run of nine $\mathrm{A}$ or $\mathrm{T}$ nucleotides is required as the minimum length to promote transcriptional rearrangement [39], and the RNA polymerase requires over seven $\mathrm{A}$ or $\mathrm{T}$ nucleotides for slippage $[41,53]$. For other IS1447-like elements that perform +1 transcriptional slippage, similar stem-loop structures can also be detected, even though they contain diverse $5^{\prime}$-codons adjacent to the slippage region compared to IS1447 (Additional file 2: Table S3). For IS1447, a modified stem-loop structure could be formed without the 3-bp upstream codon to the $\mathrm{A}_{9} \mathrm{~T}$ sequence (Fig. 4c). Thus, although the upstream RNA secondary structure may be essential for the slippage of IS1447-like elements, the $5^{\prime}$-codon adjacent to the slippage region is not indispensable.

The frameshift frequency of the IS elements could be modulated by the physiological state of the host cells [8]. Hence, the microorganisms may control the expression and combination of different functional Tpase domains [11], as well as the transposition activity of the IS elements [56], by programmed frameshifting at either the transcriptional or translational level. For IS1447, transposition activity was only observed in C. thermocellum with the presence of exotic stress. For example, we detected the transposition of IS1447 only in the presence of counterselection stress reagent FUDR, which would cause cell death in this study. Zverlov et al. observed IS1447 transposition in the genome of $C$. thermocellum ATCC27405 under chemical mutagenetic stress induced by ethylmethanesulfonate [24]. Wilson et al. also detected the insertion of an IS3 element, which may be IS1447, in the genome of C. thermocellum DSM1313 during targeted gene deletions using the hypoxanthine phosphoribosyl transferase gene as the counterselection marker [57]. There could be transposition precedence for the active IS1447 with the presence of exotic stress. Thus, this could be an efficient method to detect the in vivo transposition and analyze the mechanism of IS1447 and other transposable elements with the presence of exotic stresses.

Clostridium thermocellum has promising industrial potential as a whole-cell catalyst to convert lignocellulose to fermentable sugars, biofuels, and biochemicals. Nevertheless, targeted engineering is still required to enhance its degrading activity and the yield of target products [26, $27,31]$. Because the transposition of IS1447 may make genetic manipulation difficult, it is necessary to inactivate IS1447 in C. thermocellum. However, it would be difficult or tedious work to delete fifteen highly identical copies of IS1447 genes in C. thermocellum DSM1313. However, IS1447 elements may be silenced or blocked if its inducing activation mechanism could be identified. Thus, future investigations of the transposition and activation 
mechanism of IS1447 will provide valuable information that will enhance our understanding of these intriguing and potentially useful systems.

\section{Conclusions}

Insertion sequences are of great interest in developing transposon-based tools for genome characterization, mutagenesis, and editing. Hence, the sequence features and transposing mechanisms should be extensively understood. Thermophilic IS elements are rarely reported compared to mesophilic ones. Here, we discovered and analyzed an active IS element IS1447 from a thermophilic bacterium $C$. thermocellum, representing a novel Firmicutes-enriched subgroup of the IS3 family. Interestingly, the well-known IS3-family members usually employ -1 ribosomal frameshifting for the transposase expression, but IS1447 exhibits + 1 transcriptional slippage within a region of seven successive A nucleotides, although it is generally considered that RNA polymerase needs more than seven A or T nucleotides for slippage. IS1447-subgroup elements are abundant in the genomes of several lignocellulosic bacteria. Thus, the investigation of IS1447 will enrich our understanding of the transposition behavior of IS elements and may promote the development of IS-based mutagenesis tools for thermophiles.

\section{Methods}

\section{Bacterial strains and cultivation}

Bacterial strains used in this study are listed in Additional file 1: Table S2. Escherichia coli strains were cultivated aerobically at $37^{\circ} \mathrm{C}$ in Luria-Bertani (LB) liquid medium with shaking at $200 \mathrm{rpm}$ or on solid LB plate with $1.5 \%$ agar. C. thermocellum strains were grown anaerobically at $55{ }^{\circ} \mathrm{C}$ in $\mathrm{MJ}$ medium [58] with $5 \mathrm{~g} / \mathrm{L}$ cellobiose as the carbon source. $30 \mu \mathrm{g} / \mathrm{mL}$ chloramphenicol and $100 \mu \mathrm{g} /$ $\mathrm{mL}$ ampicillin were supplemented to the medium when necessary.

\section{Phylogenetic analysis}

Phylogenetic analyses were performed with the MEGA5 software (version 5.05, [59]). The nucleotide sequences encoding the IS1447-like OrfAB proteins (Additional file 2: Table S4) were retrieved from NCBI, translated in silico, and pre-aligned with the ClustalW algorithm. For proteins that require rearrangement for full-length expression, artificial fusion was performed by manually deleting one nucleotide $\mathrm{A}$ from the $\mathrm{A}_{9} \mathrm{~T}$ frameshift window without changing the OrfAB amino acid sequence. The full-length OrfAB-like proteins were then aligned with the ClustalW algorithm, and the alignment was refined manually. The DNA sequences were aligned according to the aligned proteins. Phylogenetic trees were calculated based on amino acid sequences of full-length OrfAB-like proteins using the neighborjoining algorithm [60]. Tree topologies were verified by bootstrap analysis with 1000 replicates. The aligned DNA sequences were analyzed by WebLogo [43] to show the consensus sequences. The affinitions of the organisms containing the IS1447_OrfAB-like proteins were identified using the Taxonomy Browser (https://www.ncbi.nlm. nih.gov/taxonomy).

\section{Nucleic acid isolation and reverse transcription PCR} Clostridium thermocellum DSM1313 was grown at $55^{\circ} \mathrm{C}$ to mid-log phase with $5 \mathrm{~g} / \mathrm{L}$ cellobiose as the carbon source. Genomic DNA and total RNA were isolated using Blood \& Cell Culture DNA Mini Kit and RNeasy Mini kit (Qiagen), respectively. Reverse transcription was performed using the isolated total RNA as the template with SuperScript III First-Strand Synthesis Supermix (Invitrogen) and random hexamer primers. Both genomic DNA and cDNA were used as templates for PCR with $p f u$ DNA polymerase and primer set OrfAB-1/-3 (Additional file 1: Table S1). The isolated RNA was also used for PCR to test the potential contamination of DNA.

\section{Cloning and sequencing}

The obtained PCR products were purified using a Gel extraction Kit (Omega), ligated to a pMD19-T vector (Takara), and transformed into E. coli DH5 $\alpha$ competent cells according to the manufacturer's protocol. Thirty and fifty-two colonies were randomly selected from the pools derived from genomic DNA or cDNA, respectively, for sequencing using a universal M13F primer.

\section{Plasmid construction}

All plasmids were constructed based on pET21a (Additional file 1: Table S2) to add a $3^{\prime}$ terminal successive six histidine tag to the target proteins for further purification and immunoblotting. All segments of IS1447 were amplified from the genome DNA of C. thermocellum DSM1313 and verified by sequencing. Restriction sites $\mathrm{XbaI}$ and XhoI were used for DNA cloning to eliminate the ribosome-binding site (RBS) of pET21a. Instead, the potential endogenous RBSs of IS1447 was employed for protein translation. Site-directed mutagenesis was accomplished using the reverse PCRs according to a previous report [61]. To construct plasmids pET21a-OrfAB-eGFP and pET21a-OrfAB-A $A_{8}$ eGFP for the fused expression of eGFP with OrfAB and OrfAB- $\mathrm{A}_{8}$, respectively, primer set 21-r1/2 was used to linearize pET21a-OrfAB or pET21aOrfAB- $\mathrm{A}_{8}$ through PCR first. The eGFP gene was then amplified by eGFP-o1/2. The primers $21-\mathrm{r} 1$ and $21-\mathrm{r} 2$ contained overlapping regions of eGFP-o1 and eGFP-o2, respectively, and seamless assembly cloning was further performed to ligate the linear plasmids and eGFP genes 
according to the manufacturer's protocol (Clone Smarter Technologies).

\section{Protein expression}

The pET21a-derived plasmids were constructed in E. coli DH5 $\alpha$, and then transformed into E. coli BL21(DE3) for protein expression. The cells were cultivated to the midexponential phase $\left(\mathrm{OD}_{600} \mathrm{~nm}=0.8-1.0\right)$, and $1 \mathrm{mM}$ of isopropyl $\beta$-D-thiogalactoside (IPTG) was added to initiate the protein expression. The cells were further cultivated at $30{ }^{\circ} \mathrm{C}$ for $3 \mathrm{~h}$ and were used for fluorescence imaging with a fluorescent microscope BX51 (Olympus, Beijing, China) or for sodium dodecyl sulfate-polyacrylamide gel electrophoresis (SDS-PAGE) analysis.

\section{Protein analyses}

SDS-PAGE was performed to check the protein purity and composition as previously described [62]. The molecular weight of the protein was estimated according to the relative mobility of protein ladders $(11-116 \mathrm{kDa}$ or $10-230 \mathrm{kDa}$, New England BioLabs). The Bradford method was used for protein quantification [63]. The mass spectroscopy analyses were achieved using MaldiTOF-TOF (Sangon Biotech). For immunoblotting, the SDS-polyacrylamide gel was wet blotted onto a presoaked polyvinylidene difluoride membrane at $400 \mathrm{~mA}$ for $1 \mathrm{~h}$ in an ice bath. The membrane was then blocked by incubating overnight in TBST buffer $(20 \mathrm{mM}$ Tris$\mathrm{HCl}, 138 \mathrm{mM} \mathrm{NaCl}, 0.08 \%$ Tween 20, $\mathrm{pH}$ 7.6) containing $50 \mathrm{~g} / \mathrm{L}$ skim milk. Afterward, the membrane was incubated for $2 \mathrm{~h}$ at room temperature with anti-His6tag rabbit IgG according to the manufacturer's protocol (Sangon Biotech). Then, the membrane was washed three times with TBST buffer, incubated for a further $2 \mathrm{~h}$ with a solution of anti-rabbit IgG(Fc) goat IgG conjugated with alkaline phosphatase (Sangon Biotech), washed as before, and visualized using HyGlo HRP ECL Detection Kit (MDBio) in accordance with manufacturers' protocol.

\section{Additional files}

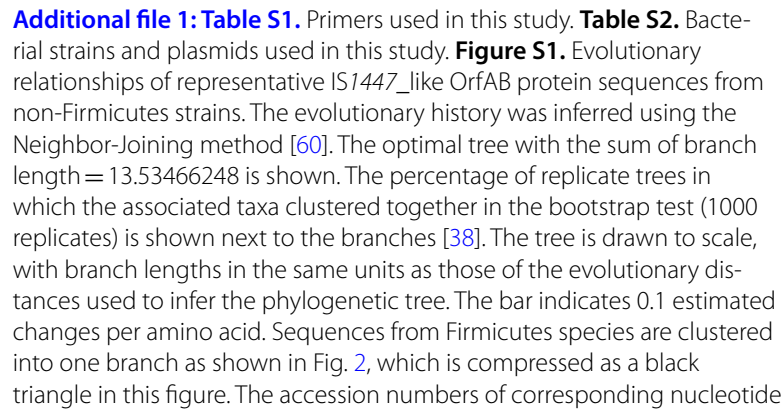

sequences are given in front of the species names. The strains with OrfAB proteins that are translated via no or -1 frameshifting are marked by an asterisk or pound sign, respectively. Figure $\mathbf{S 2}$. Sequencing results of the colonies containing partial IS1447 sequences amplified from CDNA of $C$. thermocellum DSM1313. The potential slippage-prone regions with -1 , +1 or no transcriptional rearrangement are yellow highlighted. Figure S3. Identification of OrfABt protein by mass spectrometry analysis. The protein produced by BL21(DE3)::pET21a-OrfABt- $\mathrm{A}_{8}$ with the size of $\sim 18.8$ $\mathrm{kDa}$ was investigated (Fig. 5b). Peptides detected by mass spectrometry are in red.

Additional file 2: Table S3. The sequence information of the IS1447-like genes that perform +1 transcriptional slippage. Table S4. The nucleotide sequences encoding the IS1447-like OrfAB proteins for phylogenetic analyses.

\section{Authors' contributions}

YJL, YF, and QC designed the research; YJL, KQ, JZ, and CC performed the experiments; YJL analyzed the data; YJL, YF, and QC wrote the paper. All authors read and approved the final manuscript.

\section{Author details}

${ }^{1}$ CAS Key Laboratory of Biofuels, Shandong Provincial Key Laboratory of Energy Genetics, Qingdao Institute of Bioenergy and Bioprocess Technology, Chinese Academy of Sciences, Qingdao, China. ${ }^{2}$ Dalian National Laboratory for Clean Energy, Dalian, China. ${ }^{3}$ University of Chinese Academy of Sciences, Chinese Academy of Sciences, Beijing, China. ${ }^{4}$ Present Address: Department of Biosystems Engineering, Auburn University, Auburn, AL 36849, USA.

\section{Competing interests}

The authors declare that they have no competing interests.

\section{Availability of data and materials}

All data generated or analyzed during this study are included in this published article and its additional files.

\section{Consent for publication}

Not applicable.

\section{Ethics approval and consent to participate}

Not applicable.

\section{Funding}

This work was supported by the "Transformational Technologies for Clean Energy and Demonstration", Strategic Priority Research Program of the Chinese Academy of Sciences (Grant Number XDA 21060201), the National Key Technology Research and Development Program of China (Grant Number 2015BAD15B05), the National Natural Science Foundation of China (Grant Numbers 31570029, 31670735 and 31661143023), the Key Technology Research and Development Program of Shandong (Grant Number 2018GSF1160165), the Shandong Provincial Natural Science Foundation (Grant Number ZR2016CB09) and the Major Program of Shandong Provincial Natural Science Foundation (Grant Number ZR2018ZB0208).

\section{Publisher's Note}

Springer Nature remains neutral with regard to jurisdictional claims in published maps and institutional affiliations.

Received: 25 July 2018 Accepted: 27 October 2018

Published online: 01 November 2018

\section{References}

1. Aziz RK, Breitbart M, Edwards RA. Transposases are the most abundant, most ubiquitous genes in nature. Nucleic Acids Res. 2010;38:4207-17. 
2. Nowacki M, Higgins BP, Maquilan GM, Swart EC, Doak TG, Landweber LF. A functional role for transposases in a large eukaryotic genome. Science. 2009;324:935-8.

3. Jangam D, Feschotte C, Betrán E. Transposable element domestication as an adaptation to evolutionary conflicts. Trends Genet. 2017;33:817-31.

4. Siguier P, Gourbeyre E, Chandler M. Bacterial insertion sequences: their genomic impact and diversity. FEMS Microbiol Rev. 2014;38:865-91.

5. Siguier P, Gourbeyre E, Varani A, Ton-Hoang B, Chandler M. Everyman's guide to bacterial insertion sequences. Microbiol Spectr. 2015;3:1-35.

6. Chandler M, De La Cruz F, Dyda F, Hickman AB, Moncalian G, Ton-Hoang B. Breaking and joining single-stranded DNA: the $\mathrm{HUH}$ endonuclease superfamily. Nat Rev Microbiol. 2013;11:525-38.

7. Hickman AB, Dyda F. Mechanisms of DNA transposition. Microbiol Spectr. 2015:3:1-22.

8. Chandler M, Fayet O, Rousseau P, Ton Hoang B, Duval-Valentin G. Copyout-paste-in transposition of IS911: a major transposition pathway. Microbiol Spectr. 2015;3:1-17.

9. Wagner LA, Weiss RB, Driscoll R, Dunn DS, Gesteland RF. Transcriptional slippage occurs during elongation at runs of adenine or thymine in Escherichia coli. Nucleic Acids Res. 1990;18:3529-35.

10. Atkins JF, Loughran G, Bhatt PR, Firth AE, Baranov PV. Ribosomal frameshifting and transcriptional slippage: from genetic steganography and cryptography to adventitious use. Nucleic Acids Res. 2016:44:7007-78.

11. Loot C, Turlan C, Rousseau P, Ton-Hoang B, Chandler M. A target specificity switch in 15911 transposition: the role of the OrfA protein. EMBO J. 2002:21:4172-82.

12. Siguier P, Pérochon J, Lestrade L, Mahillon J, Chandler M. ISfinder: the reference centre for bacterial insertion sequences. Nucleic Acids Res. 2006;34:D32-6.

13. Mahillon J, Chandler M. Insertion sequences. Microbiol Mol Biol Rev. 1998;62:725-74

14. Timmerman KP, Tu CP. Complete sequence of IS3. Nucleic Acids Res. 1985;13:2127-39.

15. Yamada T, Lee P-D, Kosuge T. Insertion sequence elements of Pseudomonas savastanoi: nucleotide sequence and homology with Agrobacterium tumefaciens transfer DNA. Proc Natl Acad Sci USA. 1986;83:8263-7.

16. Wood MS, Byrne A, Lessie TG. IS406 and IS407, two gene-activating insertion sequences from Pseudomonas cepacia. Gene. 1991;105:101-5.

17. Haas M, Rak B. Escherichia coli insertion sequence IS150: transposition via circular and linear intermediates. J Bacteriol. 2002;184:5833-41.

18. Prere M, Chandler M, Fayet O. Transposition in Shigella dysenteriae: isolation and analysis of IS911, a new member of the IS3 group of insertion sequences. J Bacteriol. 1990;172:4090-9.

19. Polard $P$, Prère MF, Chandler M, Fayet O. Programmed translational frameshifting and initiation at an AUU codon in gene expression of bacterial insertion sequence IS911. J Mol Biol. 1991;222:465-77.

20. Duval-Valentin G, Marty-Cointin B, Chandler M. Requirement of IS911 replication before integration defines a new bacterial transposition pathway. EMBO J. 2004;23:3897-906.

21. Loot C, Turlan C, Chandler M. Host processing of branched DNA intermediates is involved in targeted transposition of IS911. Mol Microbiol. 2004;51:385-93.

22. Chung D, Farkas J, Westpheling J. Detection of a novel active transposable element in Caldicellulosiruptor hydrothermalis and a new search for elements in this genus. J Ind Microbiol Biotechnol. 2013;40:517-21.

23. Cha M, Wang H, Chung D, Bennetzen JL, Westpheling J. Isolation and bioinformatic analysis of a novel transposable element, ISCbe4, from the hyperthermophilic bacterium, Caldicellulosiruptor bescii. J Ind Microbiol Biotechnol. 2013:40:1443-8.

24. Zverlov W, Klupp M, Krauss J, Schwarz WH. Mutations in the scaffoldin gene, cipA, of Clostridium thermocellum with impaired cellulosome formation and cellulose hydrolysis: insertions of a new transposable element, IS1447, and implications for cellulase synergism on crystalline cellulose. J Bacteriol. 2008;190:4321-7.

25. Turner P, Mamo G, Karlsson EN. Potential and utilization of thermophiles and thermostable enzymes in biorefining. Microb Cell Fact. 2007;6:9.

26. Zhang J, Liu S, Li R, Hong W, Xiao Y, Feng Y, et al. Efficient whole-cell-catalyzing cellulose saccharification using engineered Clostridium thermocellum. Biotechnol Biofuels. 2017;10:124.
27. Rydzak T, Garcia D, Stevenson DM, Sladek M, Klingeman DM, Holwerda EK, et al. Deletion of Type I glutamine synthetase deregulates nitrogen metabolism and increases ethanol production in Clostridium thermocellum. Metab Eng. 2017;41:182-91.

28. Kim S-K, Groom J, Chung D, Elkins J, Westpheling J. Expression of a heat-stable NADPH-dependent alcohol dehydrogenase from Thermoanaerobacter pseudethanolicus 39E in Clostridium thermocellum 1313 results in increased hydroxymethylfurfural resistance. Biotechnol Biofuels. 2017;10:66.

29. Biswas R, Wilson CM, Giannone RJ, Klingeman DM, Rydzak T, Shah MB, et al. Improved growth rate in Clostridium thermocellum hydrogenase mutant via perturbed sulfur metabolism. Biotechnol Biofuels. 2017;10:6.

30. Tian L, Papanek B, Olson DG, Rydzak T, Holwerda EK, Zheng T, et al. Simultaneous achievement of high ethanol yield and titer in Clostridium thermocellum. Biotechnol Biofuels. 2016;9:116.

31. Kannuchamy S, Mukund N, Saleena LM. Genetic engineering of Clostridium thermocellum DSM1313 for enhanced ethanol production. BMC Biotechnol. 2016;16(Suppl 1):34.

32. Utturkar SM, Bayer EA, Borovok I, Lamed R, Hurt RA, Land ML, et al. Application of Long Sequence Reads To Improve Genomes for Clostridium thermocellum AD2, Clostridium thermocellum LQRI, and Pelosinus fermentans R7. Genome Announc. 2016:4:e01043.

33. Argyros DA, Tripathi SA, Barrett TF, Rogers SR, Feinberg LF, Olson DG, et al. High ethanol titers from cellulose using metabolically engineered thermophilic, anaerobic microbes. Appl Environ Microbiol. 2011;77:8288-94.

34. Tripathi SA, Olson DG, Argyros DA, Miller BB, Barrett TF, Murphy DM, et al. Development of pyrF-based genetic system for targeted gene deletion in Clostridium thermocellum and creation of a pta mutant. Appl Environ Microbiol. 2010;76:6591-9.

35. Koeck DE, Zverlov W, LiebI W, Schwarz WH. Comparative genotyping of Clostridium thermocellum strains isolated from biogas plants: genetic markers and characterization of cellulolytic potential. Syst Appl Microbiol. 2014:37:311-9.

36. Rivas B, Marcobal ÁE, Gómez A, Muñoz R. Characterization of ISLpl4, a functional insertion sequence in Lactobacillus plantarum. Gene. 2005:363:202-10.

37. Petrova M, Shcherbatova N, Gorlenko Z, Mindlin S. A new subgroup of the IS3 family and properties of its representative member ISPpy1. Microbiology. 2013;159:1900-10.

38. Felsenstein J. Confidence limits on phylogenies: an approach using the bootstrap. Evolution. 1985;39:783-91.

39. Larsen B, Wills NM, Nelson C, Atkins JF, Gesteland RF. Nonlinearity in genetic decoding: homologous DNA replicase genes use alternatives of transcriptional slippage or translational frameshifting. Proc Natl Acad Sci USA. 2000;97:1683-8.

40. Baranov PV, Hammer AW, Zhou J, Gesteland RF, Atkins JF. Transcriptional slippage in bacteria: distribution in sequenced genomes and utilization in IS element gene expression. Genome Biol. 2005;6:R25.

41. Sharma V, Firth AE, Antonov I, Fayet O, Atkins JF, Borodovsky M, et al. A pilot study of bacterial genes with disrupted ORFs reveals a surprising profusion of protein sequence recoding mediated by ribosomal frameshifting and transcriptional realignment. Mol Biol Evol. 2011;28:3195-211.

42. Penno C, Sharma V, Coakley A, O'Connell Motherway M, van Sinderen D, Lubkowska $L$, et al. Productive mRNA stem loop-mediated transcriptional slippage: crucial features in common with intrinsic terminators. Proc Natl Acad Sci USA. 2015;112:E1984-93.

43. Crooks GE, Hon G, Chandonia J-M, Brenner SE. WebLogo: a sequence logo generator. Genome Res. 2004;14:1188-90.

44. Gueguen E, Rousseau P, Duval-Valentin G, Chandler M. Truncated forms of IS911 transposase downregulate transposition. Mol Microbiol. 2006:62:1102-16.

45. Lee H, Doak TG, Popodi E, Foster PL, Tang H. Insertion sequence-caused large-scale rearrangements in the genome of Escherichia coli. Nucleic Acids Res. 2016:44:7109-19.

46. Steiniger-White M, Rayment I, Reznikoff WS. Structure/function insights into Tn5 transposition. Curr Opin Struct Biol. 2004;14:50-7.

47. Nicolas E, Lambin M, Dandoy D, Galloy C, Nguyen N, Oger CA, et al. The Tn3-family of replicative transposons. Microbiol spectr. 2014;3:MDNA3-0060-2014. 
48. Sassetti CM, Boyd DH, Rubin EJ. Comprehensive identification of conditionally essential genes in mycobacteria. Proc Natl Acad Sci USA 2001;98:12712-7.

49. Copeland NG, Jenkins NA, Court DL. Recombineering: a powerful new tool for mouse functional genomics. Nat Rev Genet. 2001;2:769-79.

50. Mohr G, Hong W, Zhang J, Cui G-Z, Yang Y, Cui Q, et al. A targetron system for gene targeting in thermophiles and its application in Clostridium thermocellum. PLOS ONE. 2013;8:e69032.

51. Ewing AD. Transposable element detection from whole genome sequence data. Mob DNA. 2015;6:24.

52. Bekaert $M$, Atkins JF, Baranov PV. ARFA: a program for annotating bacterial release factor genes, including prediction of programmed ribosomal frameshifting. Bioinformatics. 2006;22:2463-5.

53. Linton MF, Pierotti V, Young SG. Reading-frame restoration with an apolipoprotein B gene frameshift mutation. Proc Natl Acad Sci USA. 1992;89:11431-5

54. Penno C, Hachani A, Biskri L, Sansonetti P, Allaoui A, Parsot C. Transcriptional slippage controls production of type III secretion apparatus components in Shigella flexneri. Mol Microbiol. 2006;62:1460-8.

55. Zuker M. Mfold web server for nucleic acid folding and hybridization prediction. Nucleic Acids Res. 2003;31:3406-15.

56. Chandler M, Fayet O. Translational frameshifting in the control of transposition in bacteria. Mol Microbiol. 1993;7:497-503.
57. Wilson CM, Klingeman DM, Schlachter C, Syed MH, Wu CW, Guss AM, et al. Lacl transcriptional regulatory networks in Clostridium thermocellum DSM1313. Appl Environ Microbiol. 2016;83:e02751.

58. Johnson EA, Madia A, Demain AL. Chemically defined minimal medium for growth of the anaerobic cellulolytic thermophile Clostridium thermocellum. Appl Environ Microbiol. 1981;41:1060-2.

59. Tamura K, Peterson D, Peterson N, Stecher G, Nei M, Kumar S. MEGA5: molecular evolutionary genetics analysis using maximum likelihood, evolutionary distance, and maximum parsimony methods. Mol Biol Evol. 2011;28:2731-9.

60. Saitou N, Nei M. The neighbor-joining method: a new method for reconstructing phylogenetic trees. Mol Biol Evol. 1987;4:406-25.

61. Hemsley A, Arnheim N, Toney MD, Cortopassi G, Galas DJ. A simple method for site-directed mutagenesis using the polymerase chain reaction. Nucleic Acids Res. 1989;17:6545-51.

62. Hong W, Zhang J, Feng Y, Mohr G, Lambowitz AM, Cui G-Z, et al. The contribution of cellulosomal scaffoldins to cellulose hydrolysis by Clostridium thermocellum analyzed by using thermotargetrons. Biotechnol Biofuels. 2014; $7: 80$.

63. Bradford MM. A rapid and sensitive method for the quantitation of microgram quantities of protein utilizing the principle of protein-dye binding. Anal Biochem. 1976;72:248-54.
Ready to submit your research? Choose BMC and benefit from:

- fast, convenient online submission

- thorough peer review by experienced researchers in your field

- rapid publication on acceptance

- support for research data, including large and complex data types

- gold Open Access which fosters wider collaboration and increased citations

- maximum visibility for your research: over $100 \mathrm{M}$ website views per year

At BMC, research is always in progress.

Learn more biomedcentral.com/submissions 